\title{
The dangers of non-powder firearms
}

\author{
Brandi A. Chrismas* and Jack Powles*
}

\begin{abstract}
Non-powder weapons have become a persistent threat in today's society. They are found outside of competitive sports at an increasing rate, being misused among young individuals, and have emerged in Canadian criminal activity. In some cases, misuse of these weapons has led to death or serious injuries. Individual and community safety are at risk when fake firearms are in the hands of criminals, as they can be altered to look and perform like real firearms. They are a particular challenge for law enforcement, who cannot be expected to distinguish fake firearms from real ones under stress. This research found fake firearms to be easily accessible and the regulations around their security and control sorely lacking and often resisted. Education regarding non-powder firearms was also found to be inadequate, when it exists at all. Awareness, education and further regulation are needed to help focus on these issues. This research concludes that it would be beneficial to treat non-powder weapons like real firearms in every aspect: storage, transportation, and handling.
\end{abstract}

Key Words Guns; replica firearms; air-powered weapons; firearms safety; community safety.

Journal of CSWB. 2019 April;4(1):13-17

www.journalcswb.ca

\section{INTRODUCTION}

Non-powder weapons gained media attention and started to enter the public consciousness in our home province of Manitoba, Canada, in 2008, following the death of 13-yearold Cody Shuya. A non-powder firearm was accidentally discharged, and the projectile pierced his eye, entering his brain. He later succumbed to his injuries ("Man charged," 2010). Since this incident, the Winnipeg Police Service (WPS) has announced that they were encountering more of these weapons on the street, a pattern found to be consistently increasing. Non-powder firearms are defined as "dangerous weapons that can inflict serious or lethal injuries and any barrelled device that does not require gun powder to launch a projectile; instead, they employ compressed air or other gases, spring or electricity to fire (includes BB guns, paintball guns, airsoft guns and pellet guns)" (WPS, 2015, p. 4). Replica firearms are "designed to resemble a real firearm" with near precision and are prohibited devices within Canada due to the danger they pose. Replica firearms can include imitation firearms (WPS, 2015, p. 5; Canada, RCMP, 2012b).

Originally created for recreation, as well as for competitive sport, these non-powder weapons are present in criminal activity, as they have been manufactured as exact replicas that look and feel real. They are described as having been modified to fire bullets, while looking identical to an actual firearm, and they can be purchased directly from a shelf display in stores. A member of law enforcement may be unable to discern between a non-powder firearm and a real firearm, and if a non-powder firearm is drawn and looks identical to an actual firearm, repercussions have the potential to be lethal.

\section{RESEARCH METHODS}

This research was first addressed in a practicum course through the University of Manitoba in the fall of 2016, where the authors partnered with the WPS for a research project that would last around six months. The WPS had already begun research on non-powder firearms, and we were engaged to undertake exploratory research, with the goal of determining the extent of issues related to non-powder firearms in Canada and future routes for research and education, as well as regulation.

Our research methodology included a literature review, scanning news, and examining policies in several police agencies. We also conducted limited primary research using both qualitative and quantitative measures. We interviewed individuals from several different law enforcement agencies, including the WPS, the Edmonton Police Service, and the Royal Canadian Mounted Police (RCMP). These individuals had first-hand experience with and expertise in non-powder weapons within their careers.

To gain the fullest picture possible, we asked both openand closed-ended questions, including:

How frequently do you come across non-powder weapons? Has the frequency increased?

Do you believe that it is difficult to distinguish a nonpowder firearm from a real firearm? 
- Have you mistaken a non-powder firearm for a real firearm in the past?

- When responding to an incident involving a non-powder firearm, how would you handle the call compared with incidents involving real firearms?

What is your opinion on the accessibility of these weapons?

- Do non-powder weapons affect community and individual safety, and if so, in what way?

- Are you aware of injuries that have occurred using non-powder firearms, and have you been injured when encountering a non-powder firearm?

What would need to change in order for there to be regulation of these weapons?

What else could be done to prevent further misuse of non-powder weapons?

We collected quantitative data from the same three law enforcement agencies regarding their policies and the number of incidents they have experienced with non-powder firearms. We also analyzed existing data, such as archival documents and news reports, to gain historical and current insight into the issue. Reports regarding injury and past use of non-powder weapons were further used to provide context. Due to limited resources because this was a class project, our findings are also limited; however, we were able to form some definitive conclusions and observations that will hopefully point the way forward for continuing research in this area.

The themes that arose throughout this research were a result of broad exploratory research to gain insight into an existing problem in Canada. This research includes insight from the perspective of law enforcement agencies and existing documentation. The framework follows a logical flow, with the introductory chapter outlining the scope and focus of the study, subsequent information gathered throughout the research, and a conclusion of the research. The themes that emerged from the interview data, specific challenges, and recommendations are identified as follows:

Characteristics of non-powder weapons

Replications of or alterations to non-powder weapons

Distinguishing velocity

Law enforcement incident response

Increased frequency of non-powder weapons

Safety and injury

Retailers and accessibility

Youth and non-powder weapons

\section{Characteristics of Non-Powder Weapons}

Non-powder firearms come in all shapes and sizes and in a multitude of colours, with a variety of ammunition, including BB-sized lead shotgun shot (aka BB's), lead pellets, gelatine paint balls, and plastic pellets. Pellet guns use small pellets of several different designs with different purposes, for example, wad cutters, sharp pointed, and round nosed. Hollow-point rounds are used for hunting and may cause maximum damage due to their increased diameter upon impact (Laraque, 2004). Laraque points out that larger calibre pellets can penetrate body mass at lower velocities due to their increased mass, and this can cause serious harm to the individual using the weapon or to the public.
Non-powder firearms include three driving force mechanisms: the spring piston (spring-air), the carbon dioxide mechanism, and the pneumatic system (air). Non-powder firearms are further defined by their "muzzle velocity" (the speed of a projectile as it leaves the muzzle of the firearm) and the "muzzle energy" (the energy of the projectile as it leaves the muzzle of the firearm) (Canada, RCMP, 2012a). Traditional firearms and non-powder firearms can have similar velocities, and Laraque (2004) explains that "the range of muzzle velocities for non-powder firearms overlaps velocities reached by traditional firearms." Rifled non-powder firearms have a higher velocity, as "the longer the gun barrel is, the higher the velocity" (Laraque, 2004).

Replications of or Alterations to Non-Powder Weapons Non-powder firearms can be altered in various ways. For example, "dieseling of the barrel" involves oil placed in the barrel providing increased projectile speed, as well as an explosion when the projectile is launched out of the barrel (Laraque, 2004). A member of the National Weapons Enforcement Support Team (NWEST) explained that non-powder firearms can have the same "functions, safeties, everything" as a real firearm, and there are kits available to make them look more realistic (personal communication, March 15, 2017).

Offenders usually opt for non-powder firearms that are similar looking to real firearms. However, Constable Roemer of the Firearms Investigative Analysis Section (FIAS) of the WPS explains that criminals frequently opt to buy the cheaper non-powder firearms that are often clear plastic and then paint them black in order to make them look similar to a real firearm (personal communication, March 30, 2017). Conversely, a real firearm may also be painted in a highlighter colour or with an orange tip in order to make it look like a non-powder firearm; this could pose a danger to police officers who may hesitate to react in lethal encounters, thinking they are facing a toy gun. A member from NWEST stated that most non-powder handguns are made of materials that are similar to real firearms, such as metal, and to the exact specifications, allowing them to be modified to fire bullets (personal communication, March 15, 2017).

\section{Distinguishing Velocity}

A member of NWEST stated that the velocity at which nonpowder firearms shoot is increasingly becoming a concern (personal communication, March 15, 2017). In 2014, the three-tier approach was created for non-powder firearms. The second tier in this approach is medium velocity, where a firearm shoots a projectile at a speed anywhere from 249 feet per second up to 499 feet per second. These weapons are able to cause injury due to increased projectile speed. The NWEST member explained that medium-velocity weapons are of importance in regard to marking and identification, since they do not require a firearms license, and, in order to identify the velocity, a chronograph would have to be used to test them or a round would have to be fired (personal communication, March 15, 2017).

\section{Law Enforcement Incident Response}

The FIAS section of the WPS is responsible for various departments, including test firing for functional examination and classification of firearms and non-powder firearms 
for court purposes. Members of FIAS reported that many of these law enforcement experts could not distinguish a non-powder firearm from a real firearm at first (personal communication, March 30, 2017). A member of NWEST explains that, just because an individual is Municipal Police or RCMP "does not mean they have extensive gun knowledge; all that you can be guaranteed of is they can use their gun that they were trained on" (personal communication, March 15, 2017). Members from the NWEST division and individuals from FIAS have explained that they have not personally mistaken a non-powder firearm for a real firearm or vice versa. However, both of these divisions described that this distinction is dependent on individual circumstances and officer experience (personal communication, March 15, 2017, and March 30, 2017).

Police are trained to treat all firearms as though they were real and loaded and not to consider whether the firearm is an airsoft gun, BB gun, pellet gun, other non-powder gun, or a real firearm. Constable Marshall from the Edmonton Police Service (EPS) explains that it is "against the grain of firearms training" to distinguish a non-powder firearm from a real firearm, as law enforcement individuals must treat all firearms as "loaded and ready to fire." He further explains that "this is done regardless of the projectile being fired and whether that is a known fact or not" (personal communication, April 2017).

Constable Ogwal of FIAS states that, by its very nature, an incident response to a firearm can occur quickly, with a perpetrator being able to draw, aim, and fire in a very short period of time (personal communication, March 30, 2017). An officer needs to formulate a response to a potentially deadly force threat; if they believe it is a firearm, the officer will respond to it like a firearm. If it is confirmed to be a non-powder firearm and the perpetrator is presenting it in a threatening manner, then police officers will also treat the situation as though that firearm were potentially a real firearm.

A member of NWEST explains that an offender may want to influence "the reaction of the officer to shoot him." This could be a reason individuals might alter the appearance of weapons, whether "[it] slows the officer down, gives him a pause, [the officer] does not pull the trigger, [it] gives [the individual] more time to engage" (personal communication, March 15, 2017). This is what is known as "suicide by cop" (WPS, 2015), where an offender will pull a non-powder weapon, or even a weapon that does not function at all, in order to get killed by law enforcement. Ontario Provincial Police forensic psychiatrist Dr. Peter Collins has found that over "one-third of all police deadly force encounters could be classified as suicide by cop," further explaining that "approximately 20 per cent would feign having a weapon in order to fool the police into shooting them," meaning that non-powder weapons or replica weapons may have been involved (2019).

\section{Increased Frequency of Non-Powder Weapons}

According to a search done with the EPS using the Edmonton Police Reporting and Occurrence System (EPROS) with the keywords "imitation gun" OR "replica gun" OR "imitation firearm" or "replica firearm" OR "BB" or "airsoft" OR "pellet" OR "paintball" OR "cap gun" OR "starter pistol" OR "toy gun," there was an increase of 438 occurrences or $37.7 \%$ from 2014 to 2015 (EPS Analysts, April 2017). Calls trend up in the summer/warmer months, generally.
It is estimated by FIAS members that about $35 \%$ to $40 \%$ of firearms they come into contact with are non-powder (personal communication, March 30, 2017). They explain that the frequency of offences related to non-powder firearms is "pretty consistent"; however, in general, firearm-related offences are increasing by a small amount, including both real firearms and non-powder firearms (personal communication, March 30, 2017). Constable Marshall from the EPS explains that he regularly encounters non-powder weapons through the course of his duties, on average about once per week (personal communication, April 2017).

A member of NWEST stated that non-powder firearms are discussed almost daily, whether it be a call about identifying a weapon as real or not or a call about the offences regarding a non-powder firearm that was encountered. They stated that, as more people are prohibited from gun ownership by the courts, the frequency of encounters involving these firearms is increasing. Their assumption may be that if a person is in possession of a non-powder firearm, they cannot be charged for simple possession. However, a person who is prohibited, of which there is an increasing number, who is in possession of a non-powder firearm will be charged (personal communication, March 15, 2017).

\section{Safety and Injury}

Constable Marshall, from the EPS, states that "anytime any weapon is seen in public, it increases the fear of its use" (personal communication, April 2017). Members of FIAS explain that, in the hands of criminals, non-powder firearms are a threat to community and individual safety. It was further explained that offenders who use non-powder weapons use them as an intimidation factor and may not actually want to fire them because that would give away the fact that they are not real firearms (personal communication, March 30, 2017).

There have been several police shootings resulting in injury that include non-powder weapons. According to a member of NWEST, 70 injuries occurred over the last two years resulting from non-powder weapons, although the extent of the injuries is unknown. Over five years, 165 injuries resulted from non-powder weapons, although the extent of the injuries is again unknown (personal communication, March 15, 2017). There have been recent instances of events where police shootings involving non-powder firearms have resulted in death. The Ontario Human Rights Commission's report from November 2018 identifies examples of death resulting from an altercation with police where the deceased were using non-powder firearms. In 2014, Daniel Clause was killed by a Toronto Police officer after the officer testified that Clause had reached and pointed a gun in the officer's direction. The weapon was later discovered to be a non-powder firearm, a pellet gun (Ontario Human Rights Commission, 2018). In 2016, Alexander Wetlaufer was shot and killed by Toronto Police officers after he did not respond to their request to lower his gun. In this case as well, the weapon was later discovered to be a non-powder firearm, a BB gun (Ontario Human Rights Commission, 2018).

\section{Retailers and Accessibility}

Non-powder weapons are easily accessible, as they can be ordered through the mail. A member of NWEST explains, "the retailer isn't assuming that they are selling a gun for 
an illegal purpose"; however, "the firearms industry aims to sell guns, and they don't care to whom" (personal communication, March 15, 2017). There are some restrictions, such as producing an ID and being 18 years of age, imposed in order to purchase a non-powder weapon, and if these requirements are not met, the store can be charged or fined or lose its business license.

A member of NWEST explains the respect for standards in the industry as inconsistent, stating, "one manufacturer may do it and the other may not" in regard to restrictions and what kind of non-powder weapons they are selling (personal communication, March 15, 2017). An operations manager with the Canadian Firearms Office, a division of the RCMP, describes a situation where retailers were selling non-powder weapons with silencers on them, which would make those prohibited weapons (personal communication, April 10, 2017). Marking regulations are now in effect as of June 2017 to put standards in place for all manufactures to comply with. This includes requiring firearms that are imported to or manufactured in Canada to be clearly engraved with information about the velocity, year of production, manufacturer name, and serial number on the firearm and not a sticker that is easily removable.

Officer Mowatt, from the Canadian Firearms Office, explains that officers should place an emphasis on understanding the different types of firearms and which models are regulated (personal communication, April 10, 2017). There are non-powder firearms, which are not regulated and can be sold to anyone over the age of 18 , and these consist of pellet guns, airsoft guns, and BB guns. The Canadian Firearms Office does not regulate these, but they do regulate replica firearms, which are made to look and feel the same as real firearms but do not discharge a projectile. Officer Mowatt stated that understanding this difference is important, as they had an issue where "blue guns," that look and feel like real guns and are used for law enforcement training, were being sold to the general public illegally (personal communication, April 10, 2017). They reiterated that these definitions are important to regulate what retailers can and cannot sell.

\section{Youth and Non-Powder Weapons}

Constable Marshall, from EPS, explains that youth are more likely than any other group to be in possession of non-powder firearms (personal communication, April 2017). A member of FIAS explains that youth with criminal backgrounds are a concern as "they try to acquire whatever they can get" (personal communication, March 30, 2017). Members of both NWEST and FIAS expressed concern about adolescents and the fact that there is the potential for them to bring nonpowder weapons to school.

\section{CONCLUSION}

This research highlights the dangers posed by non-powder firearms to law enforcement personnel and society at large. In the many cases of misuse, injury, or even death, these weapons have proven to be a problem to individuals, communities, and law enforcement. We found that, with increasing frequency, these weapons are being used improperly, for criminal purposes. The misuse of these weapons has a ripple effect throughout the community: ordinary citizens fear them, law enforcement encounter them and have to treat them as real firearms until proven otherwise, and the healthcare system must deal with the resulting injuries or deaths. Perhaps the most direct threat we found was the common use of these easily obtained weapons by criminals, who use them as real firearms to scare and rob people. The data we gathered indicates that non-powder firearms in recent years have been built and sold to look increasingly similar to real firearms; hence they are posing greater threats to law enforcement, who must treat them as real. The economics are simple: there is a market for them and manufacturers will continue producing and selling them for profit as long as they are allowed to. Government has a responsibility to regulate things that pose a threat to public safety, and this is clearly one of them. There is currently a lack of standards and regulations governing these weapons, making them easily accessible to everyone, even minors or youths.

The marking regulations that were put in effect as of June 2017 do little to reduce the high level of accessibility, and this contributes to the potentially lethal force that law enforcement personnel may have to use in response to a nonpowder firearm encounter. The ready accessibility of these firearms causes a chain reaction of sorts, as there is a duality of increased trauma that is experienced when these firearms are involved in a lethal force situation. Affected communities, as well as front-line officers, are already placed in grievous situations when a response to a firearm escalates into a lethal force event. Officers involved in these situations may encounter additional trauma upon learning that the firearm was not in fact real, whether they were forced to use lethal force or not. Victims of robberies suffer similar trauma.

All of the responding officers from NWEST, WPS, and EPS suggested that non-powder weapons should be treated as real firearms in many respects. These include safe handling and storage, transportation, education, and awareness. They also expressed that implementing a safety course regarding non-powder weapons could potentially lessen misuse of them. This safety course could be a deterrent in itself, as it would be a day-long course, and individuals would have to weigh the costs and benefits of buying a non-powder weapon and what is required of them in order to do so.

Areas for future study could include Canada-wide research into the issues surrounding non-powder weapons. Recommendations could potentially include programs for public awareness and education, deeper regulation of the sale of non-powder firearms, and stiffer penalties when they are used in crimes. The public awareness campaign that the EPS organized was well received by the public and could be a potential template to be used nationwide.

\section{ACKNOWLEDGEMENTS}

Thank you to the agencies that contributed information to this research, including the Royal Canadian Mounted Police (RCMP), the Winnipeg Police Service (WPS), the National Weapons Enforcement Support Team (NWEST), and in particular, the Firearms Investigative Analysis Section (FIAS) and the Edmonton Police Service (EPS).

\section{CONFLICT OF INTEREST DISCLOSURES}

The authors declare there are no conflicts of interest. The views expressed in this paper are those of Chrismas and Powles and not the Winnipeg Police Service.

\section{AUTHOR AFFILIATIONS}

${ }^{*}$ Faculty of Arts, University of Manitoba, Winnipeg, MB. 


\section{REFERENCES}

Canada, Royal Canadian Mounted Police. (2012a). Air Guns. Retrieved from: http://www.rcmp-grc.gc.ca/cfp-pcaf/fs-fd/air_gun-arme_air-eng.htm

Canada, Royal Canadian Mounted Police. (2012b). Replica Firearms. Retrieved from: http://www.rcmp-grc.gc.ca/cfp-pcaf/fs-fd/replica-replique-eng.htm

Collins, Peter. (2019, January 2). A look at suicide by cop in officerinvolved shootings. Blue Line Magazine. Retrieved from: https:// www.blueline.ca/opinion/behavioural-sciences/a-look-at-suicideby-cop-in-officer-involved-shootings-6067

Edmonton Police Service. (2017, April). Statistics Analysis report. Edmonton Police Service, Alberta.

Laraque, D. (2004). Injury risk of non-powder guns. Pediatrics. 114(5). 1357-1361. doi:10.1542/peds.2004-1799
Man charged in Winnipeg shooting tied to 2008 death. (2010, May 28). CBC News. Retrieved from: http://www.cbc.ca/news/ canada/manitoba/man-charged-in-winnipeg-shooting-tied-to2008-death-1.874086

Ontario Human Rights Commission. (2018, November). A collective impact: interim report on the inquiry into racial profiling and racial discrimination of black persons by the Toronto Police Service. Retrieved from: http://ohrc.on.ca/en/public-interest-inquiry-racial-profilingand-discrimination-toronto-police-service/collective-impact-interimreport-inquiry-racial-profiling-and-racial-discrimination-black\#contents

Winnipeg Police Service. (2015). Non-powder firearms report. Winnipeg Police Service, Manitoba. 